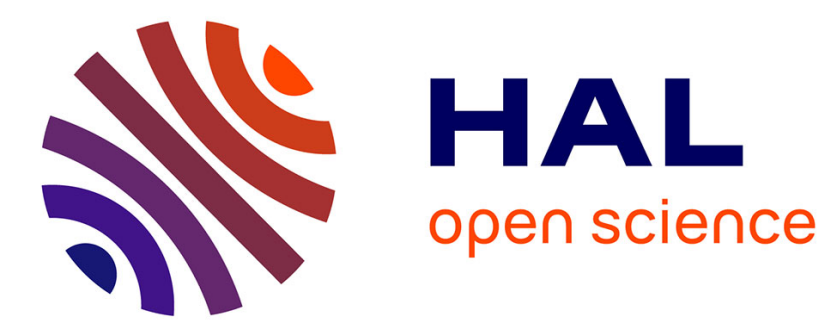

\title{
Centrale solaire et sécurité pour les navigations aériennes: photométrie photographique de miroirs d'héliostats éblouissants
}

\author{
M. A. Falipou, S. Koutchmy
}

\section{- To cite this version:}

M. A. Falipou, S. Koutchmy. Centrale solaire et sécurité pour les navigations aériennes: photométrie photographique de miroirs d'héliostats éblouissants. Revue de Physique Appliquée, 1980, 15 (2), pp.151-161. 10.1051/rphysap:01980001502015100 . jpa-00244710

HAL Id: jpa-00244710

https://hal.science/jpa-00244710

Submitted on 1 Jan 1980

HAL is a multi-disciplinary open access archive for the deposit and dissemination of scientific research documents, whether they are published or not. The documents may come from teaching and research institutions in France or abroad, or from public or private research centers.
L'archive ouverte pluridisciplinaire HAL, est destinée au dépôt et à la diffusion de documents scientifiques de niveau recherche, publiés ou non, émanant des établissements d'enseignement et de recherche français ou étrangers, des laboratoires publics ou privés. 


\title{
Centrale solaire et sécurité pour les navigations aériennes : photométrie photographique de miroirs d'héliostats éblouissants
}

\author{
M. A. Falipou et S. Koutchmy \\ Institut d'Astrophysique S.A.S., 98 bis, bd Arago, 75014 Paris, France
}

(Reçu le 25 juin 1979, révisé le 16 août 1979, accepté le 12 septembre 1979)

\begin{abstract}
Résumé. - Une expérience destinée à étudier les conditions de sécurité pour la navigation aérienne liées à l'implantation d'une centrale solaire à tour est décrite. Les faisceaux convergents issus de plusieurs héliostats solaires étaient traversés à des distances allant de 500 à $1500 \mathrm{~m}$, grâce à la mise en œuvre d'un hélicoptère. Des clichés obtenus durant la traversée ont été étudiés photométriquement, de manière à déduire les coefficients de réflexion efficaces des miroirs, la distribution des intensités sur les images, et la variation des flux lumineux durant la traversée. Les effets sur la rétine éblouie sont simulés.
\end{abstract}

\begin{abstract}
An experiment aimed to study the optical conditions in connection with air traffic security in the vicinity of a large solar energy plant is described. Light beams coming from different multi-mirror heliostats were made convergent at distances from $500 \mathrm{~m}$ up to $1500 \mathrm{~m}$. Using a helicopter, photographic sequences were obtained crossing the focal plane to study images of a heliostat field. We deduced the detailed intensity distribution over the images, the effective coefficient of reflexion of the mirrors, and the variation of the flux of light during the crossing of the focus. The effect of blinding of the retina is simulated.
\end{abstract}

1. Introduction. - Plusieurs projets de Centrales Solaires comportant un ensemble très étendu de miroirs réfléchissant les rayons solaires en direction d'un point situé au voisinage du sommet d'une tour collectrice d'énergie, ont été récemment élaborés. Le CNRS, par l'intermédiaire du PIRDES et avec la participation de l'EDF, a défini les grandes lignes de la centrale solaire expérimentale THEMIS. Aux EtatsUnis, une grande centrale solaire à tour est construite près d'Albuquerque.

Une surface terrestre étendue de quelques hectares recouverte de miroirs réfléchissant le Soleil, surtout si les faisceaux sont plus ou moins convergents, peut présenter un danger pour la navigation aérienne. Dans un rapport préliminaire, F. Sicart et B. Gentili [1] ont étudié les aspects géométriques, physiologiques et circonstanciels du problème. La déduction de données quantitatives nécessitait cependant des mesures in situ du phénomène, mesures effectuées à l'occasion d'une campagne organisée sous l'égide du PIRDES au Four Solaire d'Odeillo. Plusieurs équipes ont participé à cette expérience, notamment une équipe de l'EDF, de l'INAG, de l'IGN, de l'IAP et du G. L. Odeillo. En effet, cette expérience a demandé la mise en place d'un système logistique complexe, dû à l'utilisation d'un moyen Hélicoptère comme plate-forme d'observation.
L'étude des conditions d'éblouissement par la méthode de photométrie photographique des miroirs d'héliostats a posé en outre un problème particulier de prise de vue. Nous avons essayé de résoudre ce problème sans développer aucune technologie sophistiquée particulière à cause des moyens financiers limités du budget alloué. Cependant, compte tenu de l'aspect extrêmement original de cette expérience, le maximum de matériaux observationnels (films, clichés, enregistrements) a pu être réuni, y compris des prises de vue en $16 \mathrm{~mm}$ pour le montage d'un petit film qui, outre son aspect scientifique se rapportant directement au sujet traité, possède des qualités artistiques et promotionnelles certaines. Nous n'en parlerons que très peu, dans la mesure où ce film ne présente apparemment qu'un intérêt limité pour la déduction des données photométriques quantitatives.

\section{Description de l'expérience photographique. -} Des essais préliminaires effectués en février et avril 1978 ont permis de sélectionner les paramètres instrumentaux. Pour l'obtention des clichés, nous avons utilisé :

- Chambre photographique : NIKON F $35 \mathrm{~mm}$, équipé d'un dos motorisé 250 vues. 
- Objectif : $F=300 \mathrm{~mm}$ ouverture 4,5 avec additif doubleur de focale.

- Film : Panatomic X développé dans HC 110.

- Dilution 1 : 30 à $19^{\circ} \mathrm{C}$ pendant $7 \mathrm{~min}$.

- Calibrage photométrique : échelon neutre 25 pas adaptable avec un sensitomètre sur la même chambre $24 \times 36$.

- Contrôle des séquences de prise de vue par enregistrement audio sur mini-cassette.

- Filtres neutres Wratten $d=2$ et $d=4$ adaptables sur l'objectif.

- Prise de vue $16 \mathrm{~mm}$ : Caméra Beaulieu en accéléré (64 images/s) objectif Angénieux $25 \mathrm{~mm}$ et $75 \mathrm{~mm}$, film Kodachrome 25 .

Pour effectuer les prises de vue en $35 \mathrm{~mm}$ à partir de la petite plate-forme aménagée par l'IGN dans l'hélicoptère (porte de côté enlevée) une colonne amortissant les vibrations a dû être conçue et réalisée à l'IAP. Son principe rappelle le fonctionnement d'un piston dont la partie supérieure doit rester statique. Cette partie comportait, sur un axe horizontal, une fourche assez large pour la fixation d'un carter-support qui comprenait l'appareil principal de prise de vue, le système occultant de visée et de guidage manuel, qui se faisait à l'aide de 2 poignées fixées de chaque côté, et la commande de prise de vue par rafale. La cadence des clichés était de 2 clichés par seconde et le temps de pose de 1/500 s. Ainsi, l'hélicoptère évoluant à la vitesse absolue de $1 \mathrm{~m} . \mathrm{s}^{-1}$, les séquences permettent donc d'effectuer 1 mesure ponctuelle tous les $50 \mathrm{~cm}$, le long de la trajectoire. Cette trajectoire était repérée grâce au système (balises, etc...) mis en place par l'INAG et l'IGN et devait, en principe, couper l'axe des faisceaux réfléchis par les héliostats, à différentes distances :
500,750 et $1000 \mathrm{~m}$. La figure 1 montre comment le champ se présentait dans le viseur fin de l'observateur. Enfin, les héliostats, au nombre limité de 12 environ, devaient être pointés de manière à faire toujours

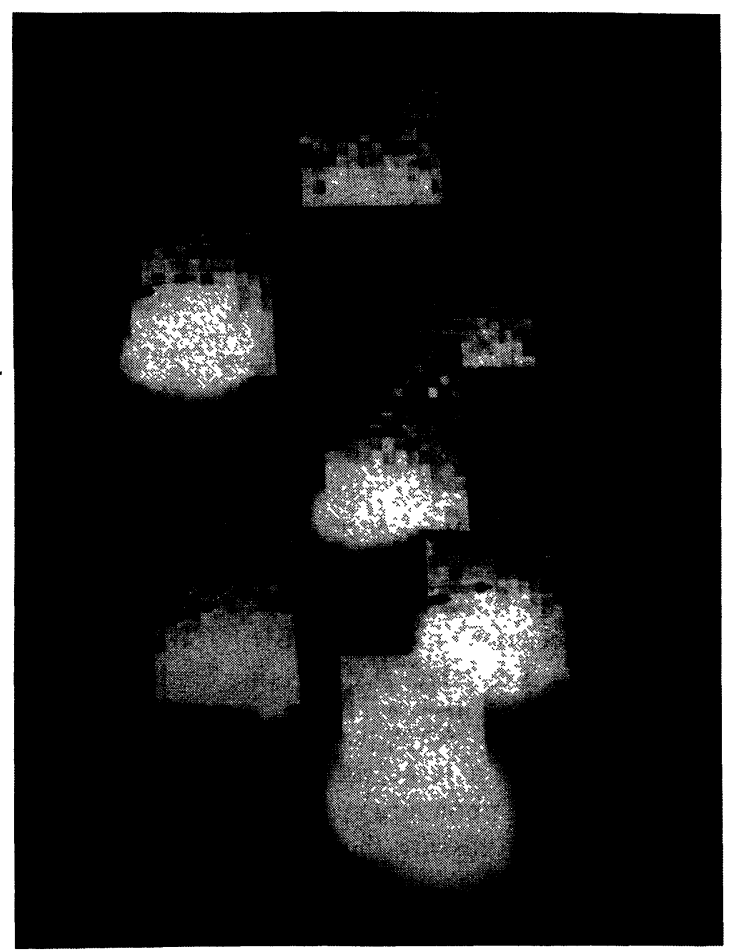

Fig. 1. - Tirage effectué à partir d'un cliché obtenu à $500 \mathrm{~m}$ de distance alors que l'observateur aborde la zone violemment éclairée dans l'espace par les faisceaux convergents. Temps de pose $1 / 500 \mathrm{~s}$; ouverture $\mathrm{F} / 8 ;$ densité neutre $d=2$.

[Picture of the illuminated heliostat mirrors obtained from a distance of $500 \mathrm{~m}$. Exposure time $=1 / 500 \mathrm{~s}$; aperture ratio $\mathrm{F} / 8$; neutral density used $d=2$.]

Tableau I. - Résumé des données concernant les différents vols en hélicoptère.

\begin{tabular}{|c|c|c|c|c|c|c|c|}
\hline Vols & Date & Focale & $\mathrm{N}$-miroirs & Navigants & H-départ & H-arrivée & Temps de vol \\
\hline - & - & - & - & - & - & - & - \\
\hline 03 & Mar 02-5 & & & Dole-Bégot & $15 \mathrm{~h} 10$ & 15 h 56 & $55^{\prime}$ \\
\hline 04 & Mer 03-5 & & & Ravaut-Bégot & & & $35^{\prime}$ \\
\hline 05 & Jeu $04-5$ & 500 & & Dole-Bégot & & & $40^{\prime}$ \\
\hline 06 & Ven $05-5$ & & & Ravaut-Bégot & 11 h 35 & 11 h 58 & $25^{\prime}$ \\
\hline 07 & Ven $05-5$ & & & Dole-Bégot & $13 \mathrm{~h} 23$ & $14 \mathrm{~h}$ & $30^{\prime}$ \\
\hline 08 & Ven $05-5$ & 500 & & Dole-Bégot & $14 \mathrm{~h} 25$ & 14 h 52 & $40^{\prime}$ \\
\hline 09 & Dim 07-5 & & & Ravaut-Koutchmy & $11 \mathrm{~h} 20$ & $12 \mathrm{~h}$ & $40^{\prime}$ \\
\hline 10 & $\operatorname{Dim} 07-5$ & 500 & 10 & Dole-Koutchmy & $14 \mathrm{~h} 25$ & $15 \mathrm{~h}$ & $35^{\prime}$ \\
\hline 11 & $\operatorname{Dim} 07-5$ & 500 & 10 & Dole-Koutchmy & $15 \mathrm{~h} 28$ & $16 \mathrm{~h} 05$ & $40^{\prime}$ \\
\hline 12 & Dim 07-5 & 500 & 10 & Dole-Bégot & 16 h 32 & $17 \mathrm{~h}$ & $30^{\prime}$ \\
\hline 13 & Mer $10-5$ & & & Dole-Pierre & $11 \mathrm{~h} 30$ & $12 \mathrm{~h} 03$ & $35^{\prime}$ \\
\hline 14 & Mer $10-5$ & 500 & & Dole-Pierre & $12 \mathrm{~h} 17$ & 12 h 45 & $25^{\prime}$ \\
\hline 15 & Mer $10-5$ & & & Calvet-Pierre & & & $15^{\prime}$ \\
\hline 16 & Mer $10-5$ & 500 & 12 & Dole-Bégot & $14 \mathrm{~h} 30$ & $15 \mathrm{~h}$ & $30^{\prime}$ \\
\hline 18 & Jeu $11-5$ & 1000 & & Dole-Bégot & $15 \mathrm{~h} 25$ & $16 \mathrm{~h}$ & $35^{\prime}$ \\
\hline 21 & Jeu $11-5$ & & & Dole-Bégot & $17 \mathrm{~h} 43$ & $18 \mathrm{~h} 15$ & $30^{\prime}$ \\
\hline
\end{tabular}


converger les faisceaux à une certaine distance choisie souvent égale à $500 \mathrm{~m}$. Le tableau I résume les données concernant chaque vol effectué pour cette expérience. Si la partie purement photographique de l'expérience, ainsi que l'exploitation quantitative des clichés par leur photométrie, ont parfaitement réussi, des difficultés sont rencontrées pour l'identification des clichés dans l'espace, c'est-à-dire pour attribuer de manière précise la valeur d'un flux mesuré sur un cliché au flux lumineux rencontré au niveau d'un certain point dans l'espace. Plusieurs raisons peuvent être invoquées pour expliquer ces difficultés :

a) Positionnement de l'hélicoptère rendu imprécis par l'effet du vent et surtout, ses inégalités. Même par très belle journée, une certaine perturbation était constatée au voisinage du faisceau traversé...

b) Imprécisions dans le pointage des héliostats. Cette imprécision est visualisable sur les clichés, d'ailleurs, et rend plus complexe l'interprétation des mesures (dispersion plus grande de l'énergie dans l'espace, etc...).

c) Pilotage aux instruments, rendu nécessaire par la configuration adoptée de l'expérience, moins précis. Un pilotage à vue aurait été préférable sans doute, mais il semblait incompatible avec un contrôle à priori des prises de vue. Enfin, un dernier point mérite d'être souligné : le déroulement de l'expérience était soumis aux impératifs de la météorologie conjugués avec un calendrier précis, d'où une contrainte au moins psychologique. Les conditions d'observation se sont avérées cependant satisfaisantes, d'où la réussite de l'expérience.

3. Description des résultats photographiques. 3.1 RéSOlution SPATIALE. - Ce problème nécessitait d'être résolu de manière sûre, sous peine de disposer d'images étalées (bougées) ne permettant pas des mesures suffisamment précises de la distribution des luminances dans les images. La figure 2 montre 2 tirages pris dans une bonne séquence, pour illustrer la résolution spatiale atteinte dans les prises de vue effectuées à $500 \mathrm{~m}$ de distance. Des détails de l'ordre de quelques $\mathrm{cm}$ sont discernables. Ceci est évidemment suffisant puisque les éléments d'héliostat font $50 \times 50 \mathrm{~cm}^{2}$ et qu'en principe, ces éléments sont homogènes. Nos clichés montrent cependant clairement des défauts de l'échelle de 3 à $5 \mathrm{~cm}$ environ. Des traces de bougé sont également discernables et certaines séquences sont même rendues inexploitables pour la déduction d'un distribution précise des intensités réfléchies. Enfin, le montage figure 3 résume suffisamment la qualité des clichés sur les séquences plein Soleil, montrant ainsi éloquemment que l'objectif principal de l'expérience photographique a été pleinement atteint.

3.2 SÉQUENCES TEMPORELLES. - La distribution des flux lumineux dans l'espace est à déduire des
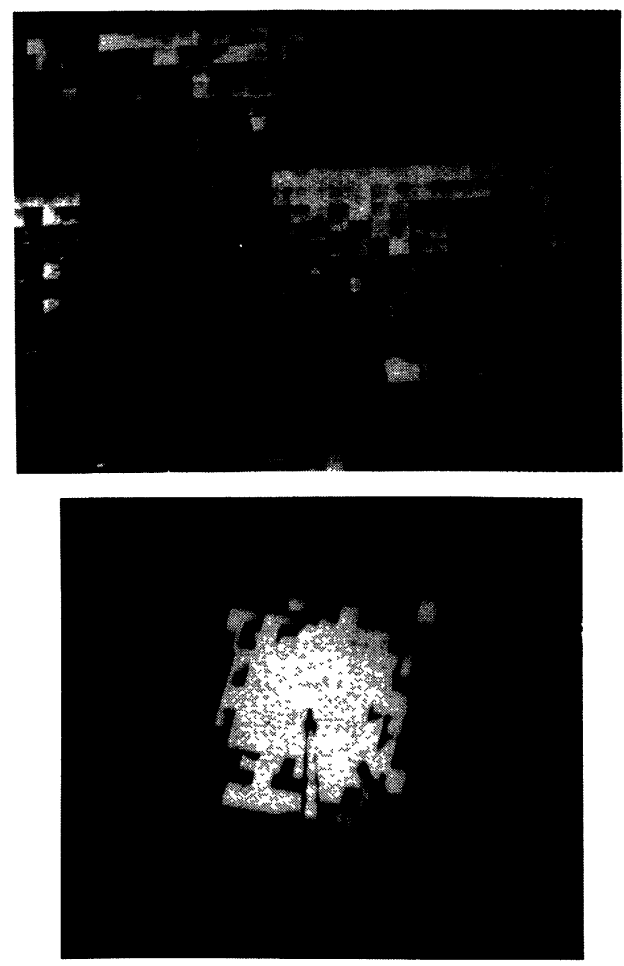

Fig. 2. - Partie supérieure : détail d'un miroir d'héliostat observé à $500 \mathrm{~m}$ de distance; partie inférieure : le même miroir éclairé plein soleil, observé avec une densité neutre $d=4$ (atténuation $10^{4}$ fois). Remarquer la netteté des images prises d'un hélicoptère au télé-objectif $f=300 \mathrm{~mm}$.

[Upper part : a heliostat mirror observed in detail at $500 \mathrm{~m}$. Lower part : the same mirror with full solar illumination attenuated by neutral density $d=4$ (attenuation $\times 10^{4}$ ). Picture obtained with a $300 \mathrm{~mm}$ Tele-lens aboard a helicopter.] a)

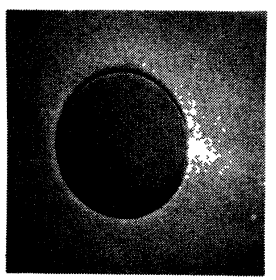

b)

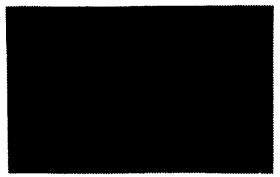

$\mathrm{d}: 500 \mathrm{~m}$

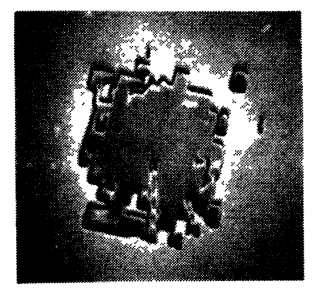

c)

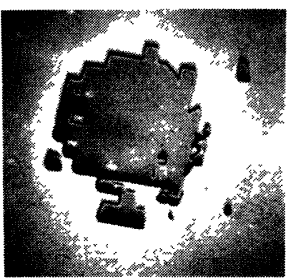

Fig. 3. - A droite, morphologie observée dans l'image du Solei réfléchie par un héliostat, sous 2 angles légèrement différents (images haut et bas de droite), pris sur un ensemble indiqué sur l'image en bas à gauche d'une distance de $500 \mathrm{~m}$. En haut à gauche, l'image du Soleil observé en visée directe, avec le même instrument, à la même échelle. Clichés analysés au Vidéo Iso Traceur (VIT) de l'IAP.

[c) and $d$ ) show the structure of the observed solar image reflected by a heliostat at two slightly different angles. Pictures taken from a set obtained at $500 \mathrm{~m}$ and shown in $b$ );a) is the directly observed solar image with the same instrument and at the same scale. The pictures were analyzed using a video-processing system.] 
séquences temporelles réalisées lors de la traversée du faisceau. Plusieurs scénarios avaient été conçus au départ, de manière à réaliser des mesures dans diverses conditions, essentiellement : 1) traversée d'un point focal réalisé par le croisement de faisceaux issus de plusieurs héliostats plans (de 8 à 12 héliostats) ; 2) traversée de ces faisceaux au double de cette distance. Le foyer principal était situé à $500 \mathrm{~m}$ des miroirs. Parmi les nombreuses séquences temporelles (voir table I) réalisées, quelques séquences seulement ont été sélectionnées pour le dépouillement, à partir des critères suivants :

1) Densités photographiques adéquates

$$
(0,3<d<2)
$$

pour les mesures microphotométriques et la déduction de la distribution des intensités grâce, en outre, à l'utilisation de clichés de calibration.

2) Séquence obtenue lors d'un passage effectué suffisamment près du point focal ou dans l'axe coupant le maximum d'intensités lumineuses.

3) Centrage correct du champ photographié sur l'ensemble (environ 20 à 25 clichés différents) des clichés par rapport au cadre $24 \times 36 \mathrm{~mm}^{2}$.

4) Etalement des images faible ou absent.

Ainsi avons-nous pu trouver 2 seules séquences (à $500 \mathrm{~m}$ et à $1000 \mathrm{~m}$ ) répondant à ces 4 critères à la fois. Les figures 4 et 5 montrent un cliché tiré de

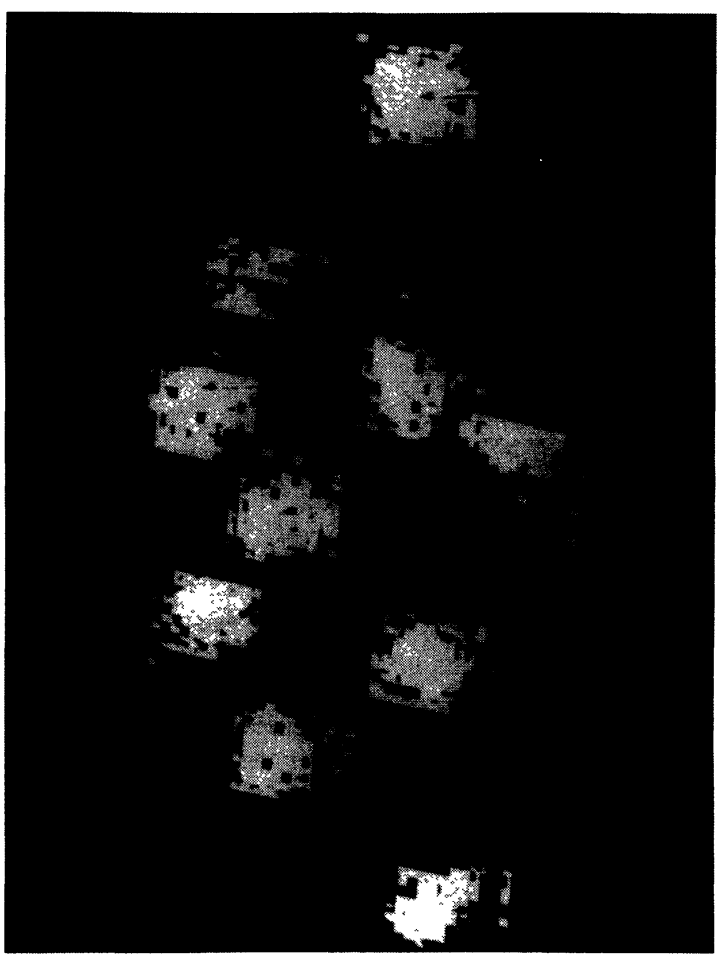

Fig. 4. - Champ d'héliostats observé en plein soleil, au voisinage du point focal, à une distance de $500 \mathrm{~m}$.

[Field of heliostats observed with full solar illumination, at a distance of $500 \mathrm{~m}$ near the focal point.]

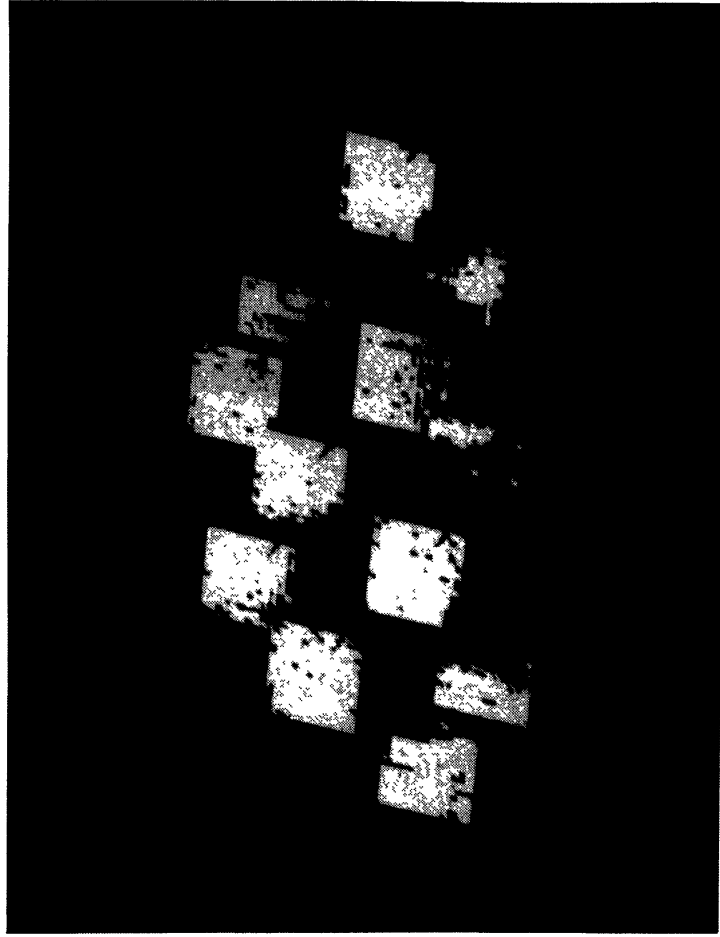

Fig. 5. - Comme sur la figure 4, mais pour une distance de $1000 \mathrm{~m}$.

[As figure 4, but for a distance of $1000 \mathrm{~m}$.]

séquences effectuées l'une à 500 l'autre à $1000 \mathrm{~m}$ où les critères 1,2 , et 4 seulement étaient réunis, d'où une qualité intrinsèque d'un seul cliché de la séquence légèrement meilleure que lors de la séquence exceptionnelle réunissant tous les critères. La figure 6 est un montage donnant la reproduction, à échelle réduite, de la séquence entière réussie à $500 \mathrm{~m}$ (au point focal) le 7 mars 78 où les 4 critères sont satisfaisants en même temps. Cette séquence a donc été tout particulièrement analysée.

3.3 ASPECT PHOTOMÉTRIQUE. - Grâce au petit sensitomètre adaptable immédiatement à la chambre photographique NIKON F, et sur la même bande de film que lors des prises de vue en vol, quelques clichés de calibrage étaient réalisés en lumière solaire diffuse à travers l'échelon, d'une part, et en visée directe sur le Soleil à l'aide des mêmes atténuateurs neutres que lors des prises de vue des miroirs, d'autre part. Ainsi, le calibrage relatif et absolu des séquences permet d'exprimer les intensités lumineuses déduites de la mesure des clichés en unité de luminance solaire. La figure 3 illustre bien le résultat principal : image du Soleil mesurée en visée directe et image du Soleil réfléchie dans les héliostats, à la même échelle, avec tous les effets géométriques produits par la disposition particulière de chaque élément d'héliostat sur la morphologie de l'image.

Pour l'étude plus détaillée de la distribution spatiale des intensités sur les images, la courbe caractéristique 


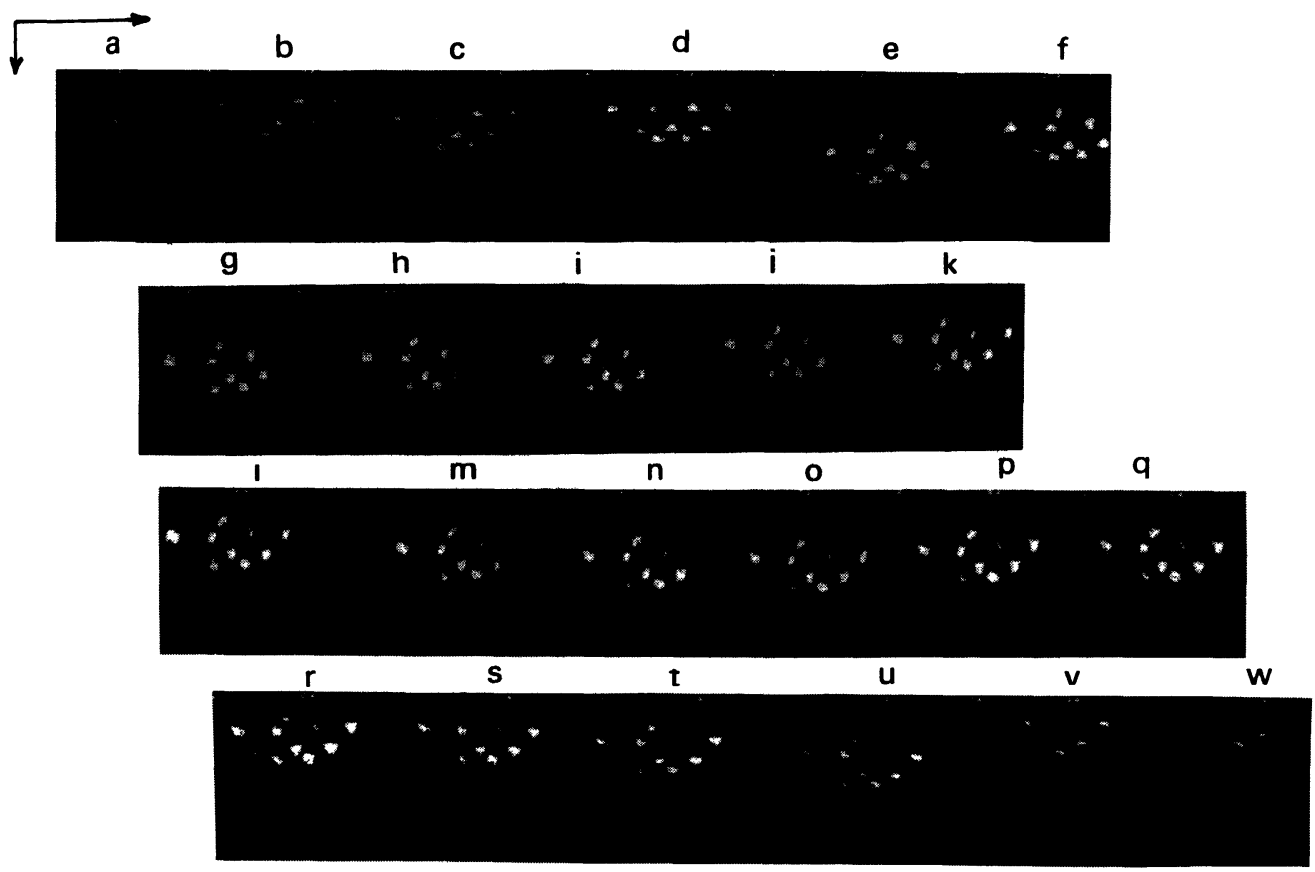

Fig. 6. - Montage montrant une séquence complète de clichés pris toutes les $0,5 \mathrm{~s}$ de temps, lors de la traversée des faisceaux focalisés à une distance de $500 \mathrm{~m}$. La progression des clichés dans le temps est de gauche à droite et de haut en bas.

[Figure showing a whole sequence of negatives take every $0.5 \mathrm{~s}$, during the crossing of the convergent beams, at $500 \mathrm{~m}$. The order is from left to right and from top to bottom.]

photographique est requise. La figure 7 donne la courbe d'étalonnage que nous avons déduite. Cette courbe est valable pour l'ensemble des clichés, car nous avons pris la précaution d'utiliser un film provenant d'une même série et de développer toujours dans les mêmes conditions. En tout cas, aucune différence sensible sur les courbes de gradation n'a pu être détectée. Comme cela avait été prévu, le facteur de contraste des clichés (valeur du gamma) est bien voisin de l'unité (voir Fig. 7).

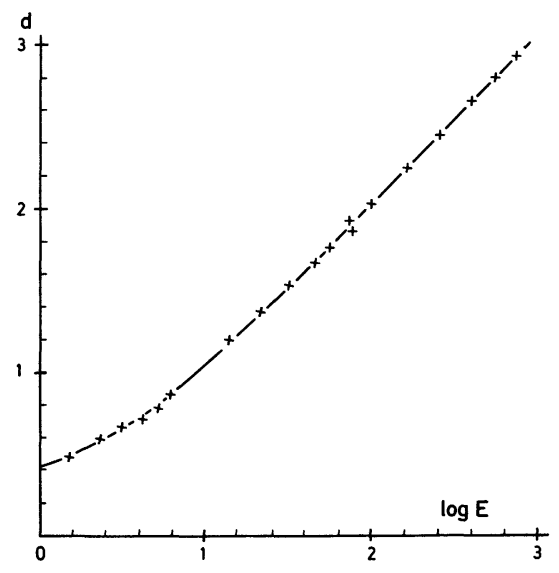

Fig. 7. - Courbe caractéristique du film utilisé pour tous les clichés $35 \mathrm{~mm}$ de l'expérience (film Panatomic $X$ développé $7 \mathrm{~min}$ dans le HC $110-$ B à $19^{\circ} \mathrm{C}$ ).

[The characteristic curve of the film used for all the $35 \mathrm{~mm}$ negatives (Panatomic X film with a development time of $7 \mathrm{~min}$ in HC 110-B at $\left.19^{\circ} \mathrm{C}\right)$.]
4. Analyse des résultats. - L'analyse microphotométrique unidimensionnelle des clichés a été effectuée à l'aide du microphotomètre Brückner, à l'Institut d'Astrophysique de Paris, alors que l'analyse bidimensionnelle a été entièrement faite à l'aide du système (prototype) de vidéo-traitement, également à l'IAP. Ce système fonctionne en mode hybride (traitement optique et électronique des images) et permet en outre des mesures très rapides de manière interactive.

4.1 ETUDE DE LA DISTRIBUTION DES INTENSITÉS SUR L'IMAGE D'UN HÉlIOSTAT A $500 \mathrm{~m}$. FACTEUR DE RÉFLEXION EFFECTIF DES MIROIRS. - Une image typique, sélectionnée sur un champ photographié lors d'un passage au voisinage du point focal à $500 \mathrm{~m}$, a été étudiée dans le détail. Cette image est repérable sur les figures 3 ou 4 (à l'extrême droite) ainsi que dans la séquence figure 6 (voir cliché no $44 \mathrm{~A}$ ), prise le 7/05/78. La distribution détaillée des intensités lumineuses sur l'image typique d'un héliostat réfléchissant dans les conditions idéales (faisceaux réfléchis autour du centre de la surface de l'héliostat) les rayons solaires, est donnée sur la figure 8 , avec 2 résolutions spatiales différentes pour rendre plus claire son interprétation. Les différents niveaux (7) des intensités sont codés avec des couleurs différentes. Le profil de la lunetteguide et son support sont parfaitement repérables dans la partie inférieure et les contours extérieurs de l'image ne rappellent que d'assez loin l'image du Soleil. Cependant, la distribution générale, mieux 

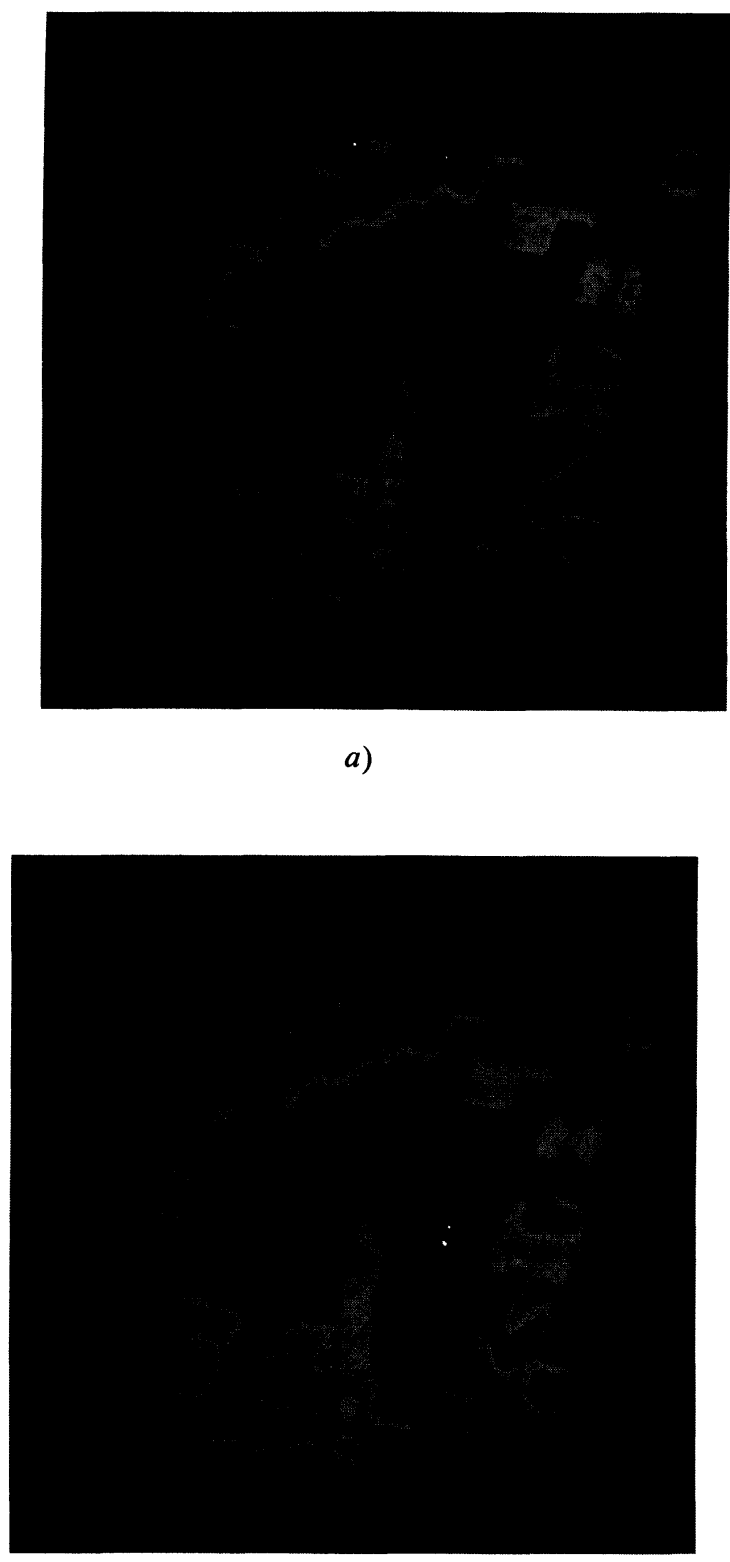

Fig. 8. - Réseau d'isophotes effectué sur une image sélectionnée d'un héliostat du champ observé figure 4. En haut, avec le maximum de résolution, en bas, en lissant le réseau pour faciliter la discrimination des niveaux. Le codage des couleurs en intensité suit la loi de la distribution spectrale de l'arc en ciel (le bleu correspond au niveau le plus intense).

[A series of isophotes obtained on a selected image from a heliostat mirror of the field observed on figure 4. Upper part : maximum resolution. Lower part : after smoothing, so as to permit the distinguishing of the levels.]

Fig. 17. - Simulation de l'effet de l'étalement de l'image d'un champ d'héliostats éclairés.

[Simulation of the smearing of the image of a field of illuminated heliostat mirrors.]

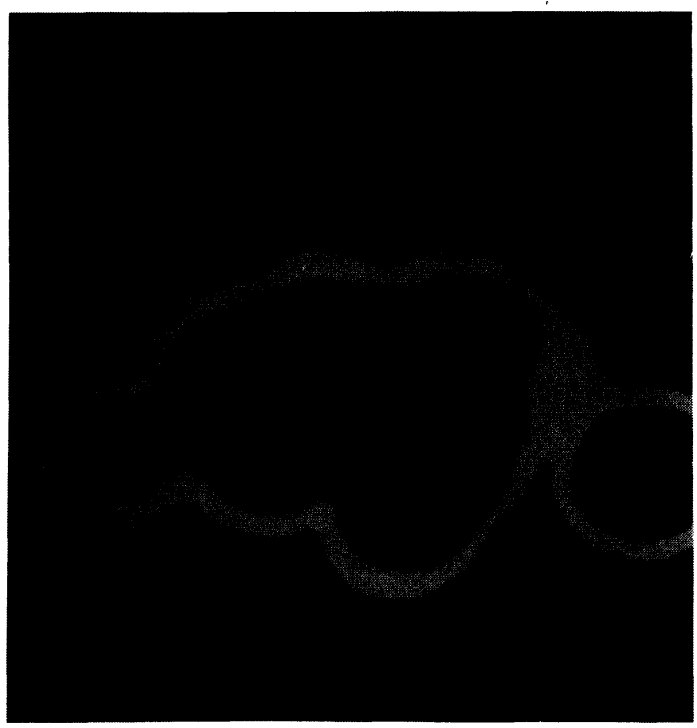


perceptible sur la partie inférieure de la figure 8 , correspond bien à celle qui aurait été vue sur l'image du Soleil, tout au moins pour ce qui est de l'effet d'assombrissement centre bord. Sur la partie supérieure, les effets sur les facettes individuelles sont bien perceptibles; on peut distinguer :

a) l'effet des interstices et espaces entre les facettes où la lumière n'est évidemment pas réfléchie,

b) l'effet produit par la diffraction de la lumière sur les bords des facettes, qui produit un étalement extrêmement réduit,

c) l'effet produit par les écarts à la planéité générale de la surface de l'ensemble de l'héliostat d'où il résulte que des facettes situées en dehors des limites de l'image solaire théorique (voir aussi la figure 3 ) se trouvent en quelque sorte éclairées.

Bien entendu, tous ces éléments entrent en ligne de compte dans la mesure de ce facteur, facette par facette, grâce à des balayages microphotométriques uni-dimensionnels effectués sur l'image. La figure 10 montre le résultat partiel d'une telle analyse lorsque les balayages sont enregistrés suivant les directions repérées sur la figure 9 qui représente toujours la même image. L'échelle des intensités a été déterminée grâce à la courbe de gradation du film, figure 7 et le calibrage en unités relatives à la luminance du disque solaire est déduit de la comparaison avec des balayages effectués sur l'image du Soleil, voir figure 11. Du fait des variations des conditions de transparence de l'atmosphère, plusieurs images du Soleil ont été étudiées et une valeur moyenne de l'intensité centrale a été déduite de l'histogramme des valeurs, voir figure 12 .

$$
I_{\mathrm{S}}(0)=220 \pm 10 \text {. }
$$

Compte tenu de l'effet de l'assombrissement centrebord, la luminance moyenne du disque est déduite

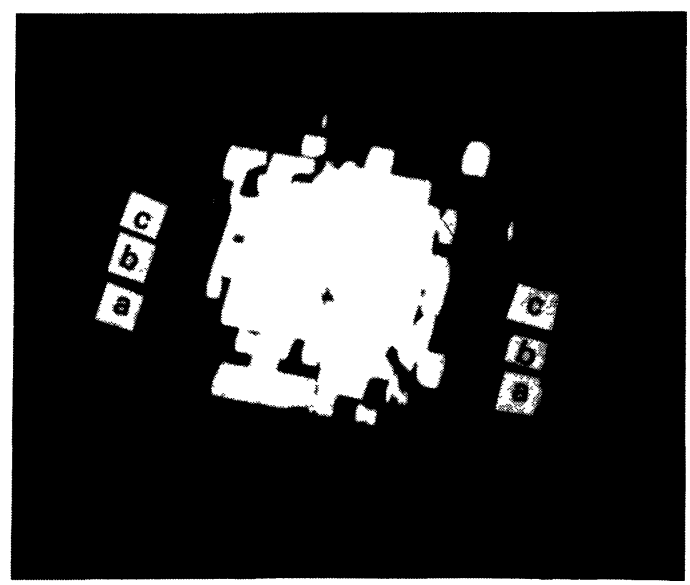

Fig. 9. - Tirage montrant l'héliostat sélectionné pour les mesures du facteur de réflexion; $a, b$ et $c$ correspondent à des coupes typiques représentées figure 10 .

[A heliostat mirror chosen to measure the reflection factor; $a$ ), $b$ ), and $c$ ) are the typical scans represented on figure 10.]

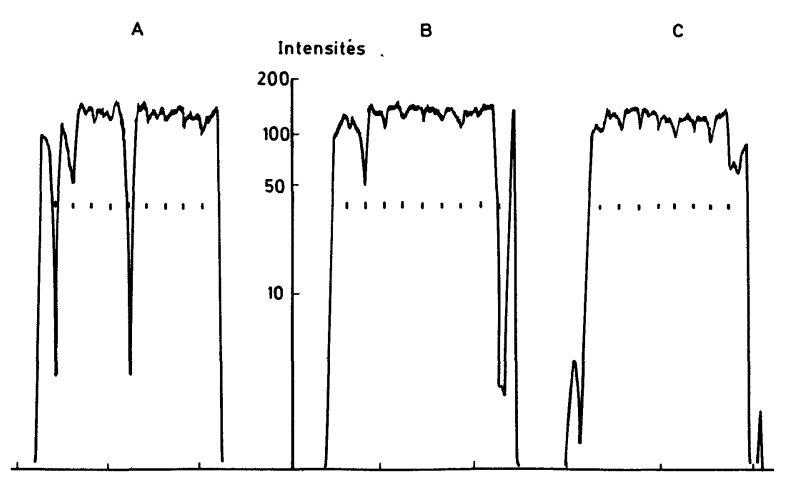

Fig. 10. - Balayages microphotométriques correspondant aux coupes indiquées figure 9 . En ordonnée, les intensités lumineuses et en abscisse, les longueurs en unité de rayon solaire ramené aux mêmes conditions d'observation.

[Microphotometer tracings corresponding to scans $a$ ), $b$ ), and $c$ ) seen on figure 9. Ordinate : intensity levels. Abscissa : lengths in solar radius unit.]

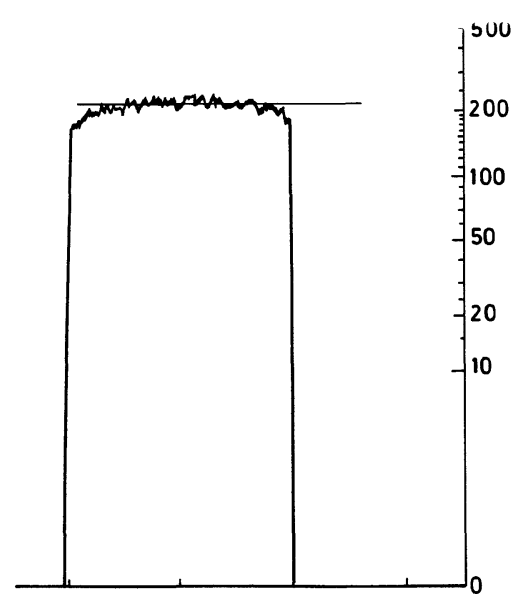

Fig. 11. - Coupe microphotométrique typique suivant un diamètre de l'image du Soleil.

[A microphotometer tracing along a diameter of the solar image.]

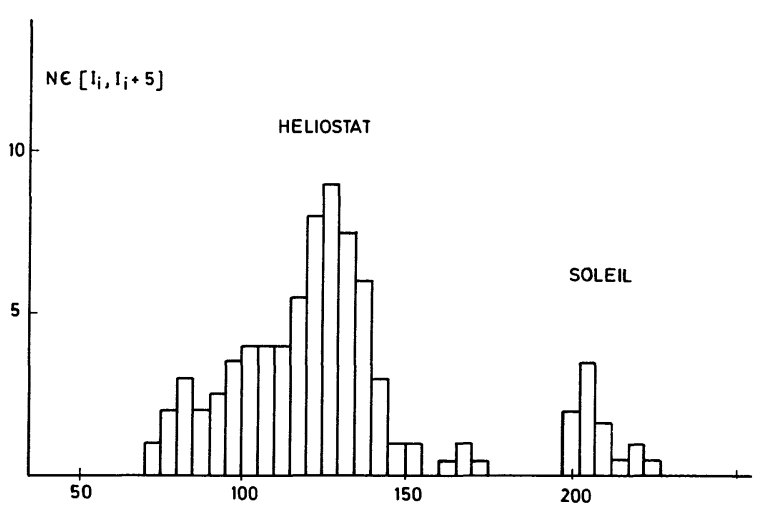

Fig. 12. - Histogramme montrant la distribution des intensités mesurées sur chaque facette ainsi que sur un certain nombre d'images $\mathrm{du}$ Soleil.

[A histogram showing dispersion of the intensity distribution over each facet of heliostat mirrors and on some of the solar images.] 
par application des facteurs bien connus à partir des mesures solaires, pour l'ensemble du spectre enregistré par l'émulsion (voir Allen, p. 169, [2])

soit ici :

$$
\bar{I}_{\mathrm{S}}=F / \pi \simeq I_{\mathrm{S}}(0) \times(1,20)^{-1}
$$

$$
\bar{I}_{\mathrm{S}}=183 \pm 8,5 .
$$

L'étude des intensités des images individuelles des facettes conduit, de la même manière, voir figure 12 , à une intensité moyenne, différente de l'intensité la plus probable, égale à :

$$
\bar{I}_{\mathrm{f}}=125 \pm \frac{15}{20} \text {. }
$$

En faisant le rapport de (4) sur (3), nous obtenons le facteur de réflexion effectif de l'héliostat pour cette distance de $500 \mathrm{~m}$ :

$$
R_{\mathrm{h}}(500) \simeq 0,68 \pm 0,11 .
$$

Soit, aussi, $68 \%$. De la même manière, nous avons étudié une image sélectionnée sur une séquence effectuée à la distance de $1000 \mathrm{~m}$, voir figure 5 , puis sur une autre effectuée à $1500 \mathrm{~m}$. Pour effectuer une comparaison quelque peu précise, nous avons préféré nous référer à des mesures faites sur des facettes sélectionnées qui fournissent les intensités maximales $I_{\mathrm{f}}^{\mathrm{M}}$ (sinon la dispersion des mesures semble trop grande pour effectuer cette comparaison), voir par exemple cet effet sur la figure 12. Nous avons obtenu les valeurs données dans le tableau II, d'où il est possible de déduire une loi empirique :

$$
\bar{I} \simeq I_{0} \times\left(d / d_{0}\right)^{-1,4}
$$

apparemment valable au moins pour l'intervalle étudié des distances. Cette loi est évidemment applicable au facteur de réflexion effectif des héliostats :

$$
R_{\mathrm{h}}(d)=R_{\mathrm{h}}(0) \times\left(d / d_{0}\right)^{-1,4}
$$

et constitue un des résultats quelque peu surprenant de cette analyse.

Tableau II. - Valeur des intensités moyennes $\bar{I}_{\mathrm{f}}$ et « maximales " $I_{\mathrm{f}}^{\mathrm{M}}$ relevées sur les balayages effectués à travers les images des facettes des héliostats observés à différentes distances $d$.

$\begin{array}{rrr}d(m) & \bar{I}_{\mathrm{f}} & I_{\mathrm{f}}^{\mathrm{M}} \\ \overline{-} & \overline{-} & \overline{175} \\ 1000 & 125 & 61 \\ 1500 & 55 & 38\end{array}$

4.2 ETUDE DE LA VARIATION DES FLUX LUMINEUX AU COURS DE LA TRAVERSÉE D'UN FAISCEAU. - L'analyse bi-dimensionnelle des images permet l'étude des variations des flux lumineux globaux sur les images consécutives d'un champ d'héliostats observé lors d'un passage à travers, par exemple, le point focal. Nous nous sommes limités à ce cas pour 2 distances différentes, 500 et $1000 \mathrm{~m}$. Le flux lumineux global enregistré sur une image est défini par l'opération :

$$
F_{\mathrm{tot}}=\sum_{i=1}^{n} \iint_{\sigma i} I_{i}(x, y) \mathrm{d} x \mathrm{~d} y
$$

la somme étant étendue sur l'ensemble des $n$ héliostats du champ et l'intégration sur la surface totale de chaque héliostat. Nous avons calculé l'intégrale dans (8) de manière approchée par la méthode des isophotes :

$$
F_{i}=\iint_{\sigma i} I_{i}(x, y) \mathrm{d} x \mathrm{~d} y \simeq \sum_{j=1}^{m} \sigma_{i, j}\left(I_{i, j}-I_{i, j-1}\right)(9)
$$

avec $\quad I_{i, 0}=0$.

Les figures 13 et 14 montrent les résultats obtenus dans la détermination des $\sum_{i} \sigma_{i, j}$ pour 2 distances différentes : 500 et $1000 \mathrm{~m}$. Les valeurs des surfaces des isophotes sont données pour 3 niveaux d'intensité dans chaque cas. Ces figures montrent, en outre, la

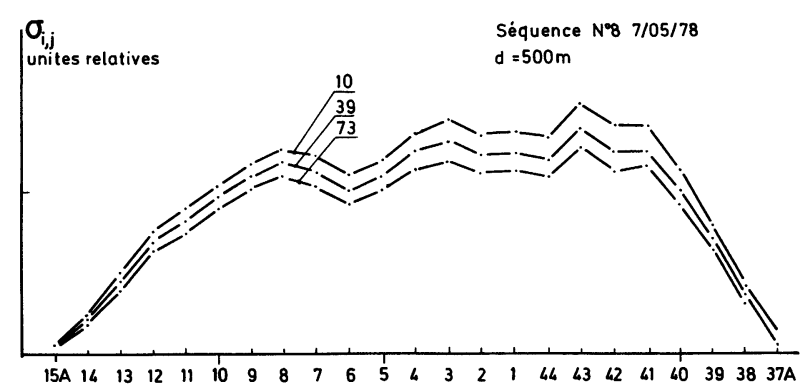

Fig. 13. - Surfaces totales mesurées des isophotes tracés sur différents clichés d'une séquence montrant un champ d'héliostats durant un passage ; chaque courbe correspond à un niveau d'intensité discret et constant pour l'ensemble de la séquence, $d=500 \mathrm{~m}$.

[Total measured areas of the isophotes drawn on different negatives of one sequence showing a field of heliostats during one passage. Each curve corresponds to an intensity level discrete and constant for the whole sequence $d=500 \mathrm{~m}$.]

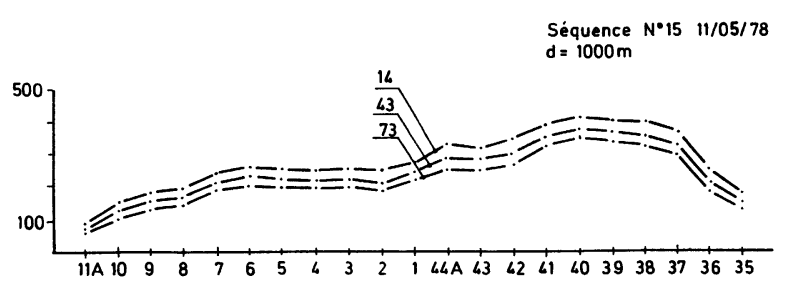

Fig. 14. - Comme pour la figure 13, mais $d=1000 \mathrm{~m}$.

[As figure 13, but for $d=1000 \mathrm{~m}$.] 
distribution de ces surfaces lors de la traversée des faisceaux. La figure 15 donne avec beaucoup plus de détails la variation des intensités en fonction de la surface d'un isophote, pour un cliché pris dans la séquence à $500 \mathrm{~m}$. Il est clair à l'examen de la figure 15 , qu'il existe une plage assez large des surfaces pour lesquelles l'intensité varie peu, ou, ce qui revient au même, que les flux lumineux sont dominés par une contribution limitée de la gamme des intensités. La figure 15 révèle également un phénomène déjà ressenti dans l'étude de l'histogramme figure 12 : il existe une petite surface sur les héliostats où l'intensité semble nettement supérieure à la moyenne.

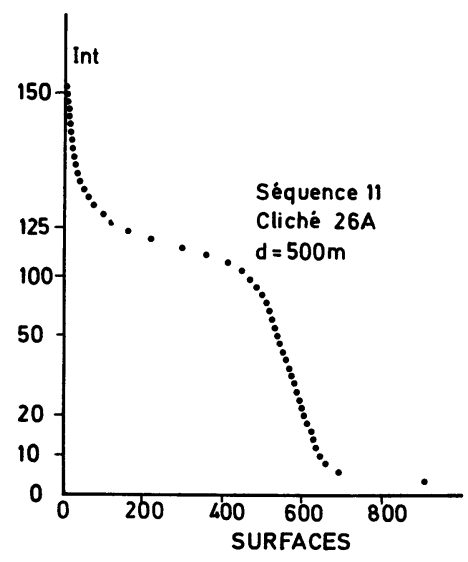

Fig. 15. - Pour un cliché du champ observé près du point focal, variation de la surface d'un isophote en fonction de son niveau d'intensité.

[Variation of the surface of one isophote versus its intensity level for one negative of the field observed near the focal point.]

Enfin, la figure 16 montre la variation des flux globaux, calculés avec la formule (9), lors de la traversée des faisceaux. L'abscisse est proportionnelle au temps. Compte tenu de la vitesse de l'hélicoptère lors de la traversée, qui était de $1 \mathrm{~m} . \mathrm{s}^{-1}$ et de la cadence des clichés, l'unité des abscisses est de $0,5 \mathrm{~m}$.

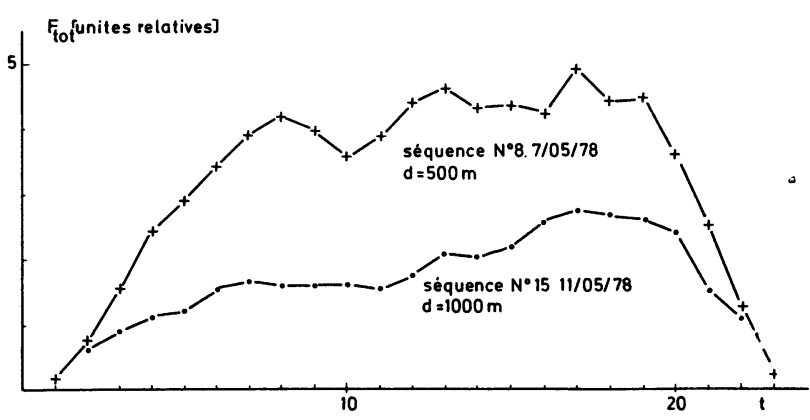

Fig. 16. - Variation du flux lumineux total produit par un champ d'héliostats durant la traversée du maximum d'intensité produit par des faisceaux focalisés, pour 2 distances typiques.

[Variation of the total luminous flux produced by a field of heliostats during the crossing of the maximum intensity due to the focusing beams, for two typical distances.]
La largeur des faisceaux est donc de l'ordre de $8 \mathrm{~m}$, ce qui semble conforme aux prédictions, compte tenu des imprécisions dont il a été question $\S 2$.

5. Conclusion. - La discussion de ces résultats prend toute son importance lorsque les données photométriques sont placées dans le contexte plus général de l'expérience [3] et surtout, en fonction des objectifs que l'on s'était fixés. Cela concerne essentiellement les effets psychophysiologiques produits sur l'œil lors de la traversée des faisceaux.

Ces effets peuvent d'ailleurs être simulés, pour ce qui est de la formation des images, grâce au traitement optique effectué sur le système de vidéo-traitement. La figure 17 montre des réseaux d'isophotes sur l'image d'un champ d'héliostats observé près du point focal, lorsque l'image est étalée à divers degrés, ce qui simule l'effet sur la rétine pour différentes positions et visions. La figure 18 montre l'évolution de l'image étalée lors d'un passage. Ce type de document permet une approche objective du phénomène d'étalement, mais il est cependant insuffisant pour pouvoir répondre de manière quantitative à la question concernant le danger présenté par l'éblouissement par les héliostats. A côté des données physiologiques qui sont fournies dans les expériences de laboratoire sur l'œil, la simulation numérique répond en partie à cette question (voir [1] et [3]).

Nous avons cependant mis en évidence plusieurs phénomènes :

a) Le facteur de réflexion effectif des miroirs dans les conditions d'éblouissement est plus faible que les valeurs théoriques et surtout, la variation de la distribution du flux lumineux avec la distance aux miroirs est plus grande que prévu. L'énergie semble se dissiper dans l'espace plus rapidement, ce qui pourrait s'expliquer par les effets produits par la diffraction sur les bords des nombreux miroirs élémentaires constituant chaque héliostat, ainsi que par la diffusion de la lumière sur les poussières et gouttelettes accumulées sur les miroirs et le long du trajet optique.

b) La traversée des faisceaux montre que l'énergie est plutôt étalée dans l'espace sur une certaine largeur qui varie avec la distance aux miroirs. L'effet d'éblouissement est donc relativement prolongé sans qu'il y ait un maximum prononcé. Enfin, si l'observateur n'accommode pas sur l'image des miroirs éblouissants, ces images seront étalées sur la rétine et l'énergie sera mieux dissipée par l'œil.

A notre avis, les deux effets mentionnés semblent réduire le degré d'éblouissement sur l'œil. Nous pouvons d'ailleurs ajouter avec certitude, pour l'avoir expérimenté nous-mêmes, que dans les conditions décrites de nos mesures, l'effet produit était soutenable et n'a pas provoqué, en aucune occasion, de lésion ou autre dommage irréversible. Il est cependant certain qu'il faut prendre en considération dans l'ave- 

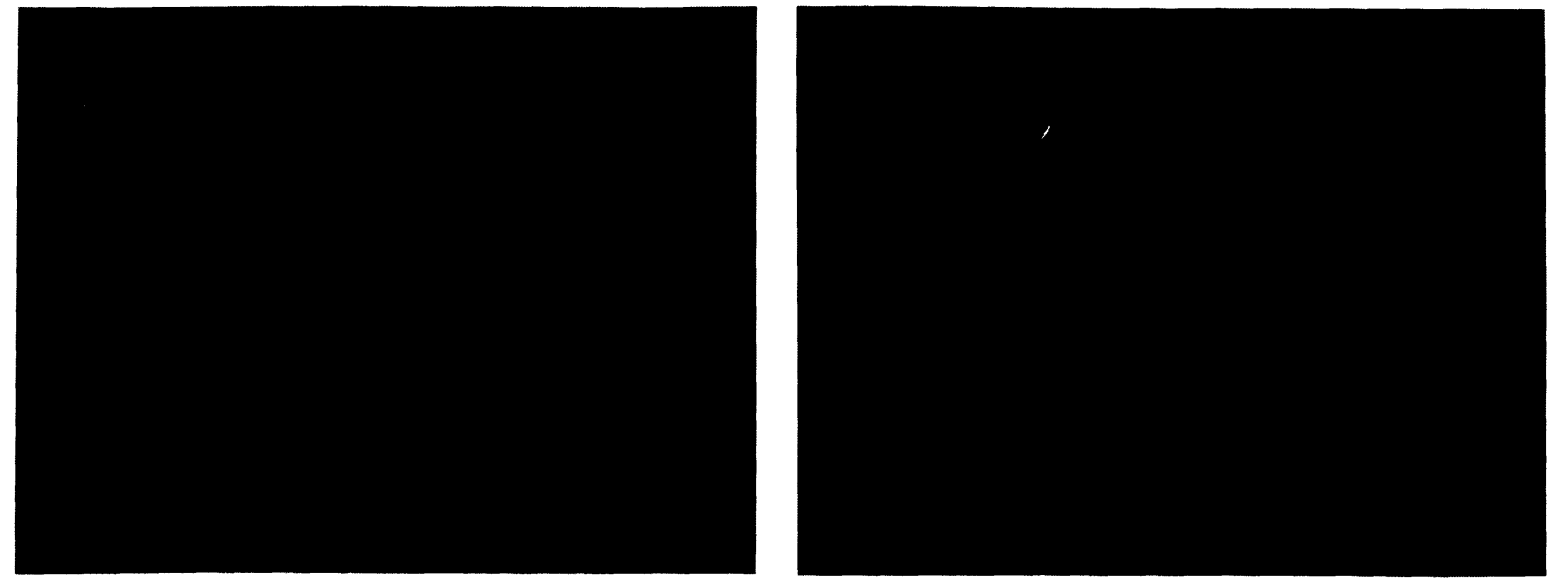

g)
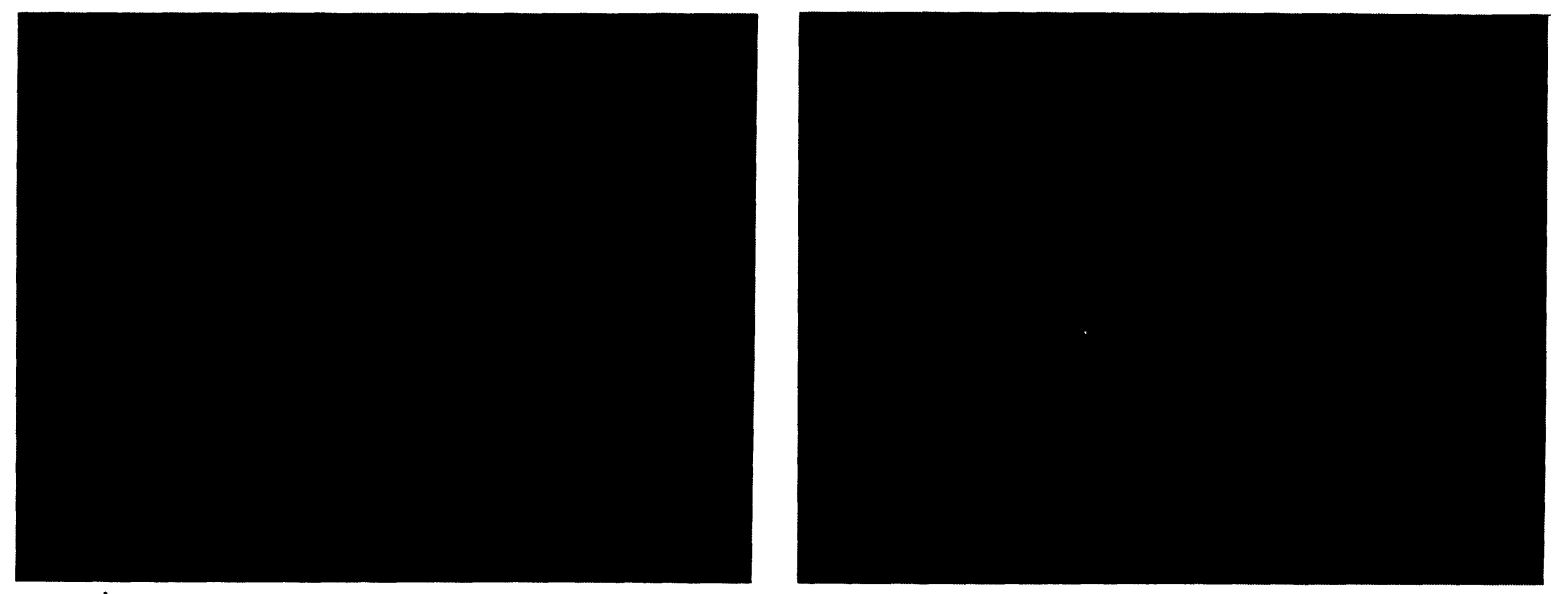

c)

f)

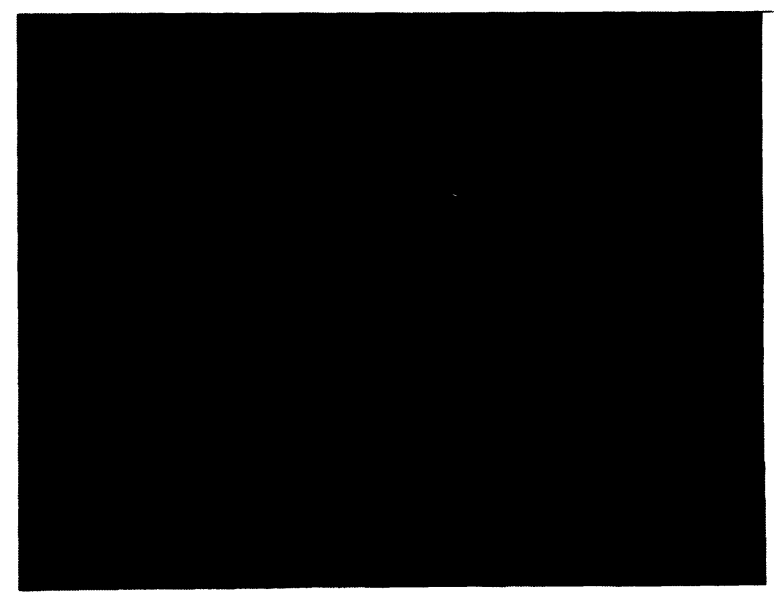

b)

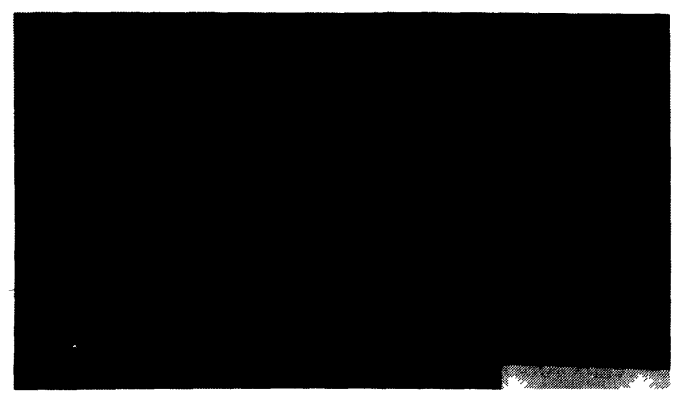

a)

Fig. 18. - Champ d'héliostats visualisé avec étalement (en bas à droite sans étalement) pour différentes positions dans le faisceau. [Smeared images of illuminated heliostat mirrors for different points across the reflected beam.] 
nir pour éviter les accidents, l'effet de surprise d'une part, l'attirance de l'œil vers quelque chose qui brille d'autre part. La projection du film qui a pu être réalisé à l'occasion de cette campagne d'essais (film disponible grâce au concours du Service des Relations Extérieures du CNRS) devrait suffire d'ailleurs pour dissuader les pilotes d'avions appelés à survoler les régions d'implantation de Centrales Solaires.

Pour ce qui est des effets physiologiques, les phénomènes mentionnés plus haut peuvent maintenant être pris en compte dans les simulations numériques (détermination des seuils critiques de lésion permanente par brûlure de la rétine). Il est clair cependant, qu'à condition de ne pas fixer les images éblouissantes, ni d'accommoder, le danger de lésion est très limité dans les conditions courantes de survol des futures centrales.

Remerciements. - Cette expérience a nécessité le concours de nombreuses personnes qui ont toute notre reconnaissance, mais nous tenons à citer tout particulièrement l'équipage de l'hélicoptère $\mathbf{M M}$. d'Arnaudy et Pierre de l'IGN et MM. Sicard et Gentili de l'EDF, qui ont assuré respectivement la coordination de toute l'opération et le repérage au sol. Enfin, nous tenons à associer Mr. J. Bégot, de l'IAP, aux résultats présentés, pour sa contribution déterminante à l'expérience et nous remercions $\mathrm{Mr}$. Etiévant pour le soutien constant qu'il a bien voulu nous apporter.

\section{Bibliographie}

[1] Sicart, F., Gentili, B., FST/ADu, THEM 77-41 (1977).

[2] Allen, C. W., Astrophysical Quantities, 3rd Edition (The Athlone Press) 1973.

[3] Brumleve, T. D., Experimental Assessment of Eye Hazards associated with Central Receiver Heliostat Arrays, Prepint (1978). 